\title{
I doni di Cadmo - e per Cadmo
}

Les dons de Cadmos - et Pour Cadmos

Cadmus and His Gifts

\section{Annamaria Peri}

\section{(2) OpenEdition}

\section{Journals}

Edizione digitale

URL: https://journals.openedition.org/gaia/331

DOI: 10.4000/gaia.331

ISSN: 2275-4776

\section{Editore}

UGA Éditions/Université Grenoble Alpes

\section{Edizione cartacea}

ISBN: 978-2-37747-057-0

ISSN: 1287-3349

\section{Notizia bibliografica digitale}

Annamaria Peri, «l doni di Cadmo - e per Cadmo», Gaia [Online], 21 | 2018, online dal 01 novembre 2018, consultato il 09 décembre 2021. URL: http://journals.openedition.org/gaia/331 ; DOI: https:// doi.org/10.4000/gaia.331

Questo documento è stato generato automaticamente il 9 décembre 2021.

Gaia. Revue interdisciplinaire sur la Grèce archaïque 


\title{
I doni di Cadmo - e per Cadmo
}

\author{
Les dons de Cadmos - et Pour Cadmos \\ Cadmus and His Gifts
}

Annamaria Peri

\section{Introduzione}

1 Lo straniero civilizzatore fa doni alla terra che lo accoglie. La risolleva da mostri o tiranni, la provvede di risorse, istituisce culti, importa tecniche e sapere. Cadmo, fenicio secondo una tradizione, greco di stirpe ma proveniente dalla Fenicia o dall'Egitto secondo un'altra ${ }^{1}$, libera il suolo della futura Tebe dal drago divoratore che stava a guardia di una fonte (la fonte di Ares), e così facendo rende attingibile a uomini e animali il bene vitale dell'acqua ${ }^{2}$; poi fonda la città, la popola; e già prima, nelle stazioni del suo peregrinare dall'Oriente fino al cuore della Grecia, aveva istituito culti ${ }^{3}$ e disseminato l'uso dell'alfabeto ${ }^{4}$.

2 Ma a Cadmo, allo straniero, che cosa dona la terra ospite? La domanda, trascritta in termini di storia della cultura, potrebbe porsi in questo modo: se il mito di Cadmo esprime la memoria e la gratitudine di un popolo che ha beneficiato dei contatti con una florida civiltà del Vicino Oriente ${ }^{5}$, che cosa avvertivano i Greci di aver saputo offrire a loro volta allo straniero? Perché è legittimo supporre che nel processo di compenetrazione di due civiltà, per quanto diverso il loro grado di sviluppo, il trapasso di elementi culturali non avvenga tutto in un'unica direzione. I dati dell'archeologia, anzi, ci suggeriscono precisamente il contrario: parlano di un contributo talora sostanziale di Tebe in età protostorica agli equilibri politici del Vicino Oriente ${ }^{6}$; attestano la perizia degli artigiani cadmei nella cesellatura dell'avorio che giungeva dall'Est o dall'Africa settentrionale ${ }^{7}$. $E$ dunque: vi sono, nel mito, tracce di aspirazioni civilizzatrici da parte greca?

3 La leggenda di Cadmo si presta magnificamente a questa interrogazione, per almeno due motivi. Il primo è che ci pone dinanzi agli occhi un caso di osmosi particolarmente intensa fra elementi forestieri ed elementi nativi, fra l'allotrio e l'autoctono: lo 
straniero che sparge nella terra locale i denti di una creatura mostruosa locale, e ne vede scaturire in armi il popolo di cui si farà re, è come l'incarnazione di una forza esterna che viene a depurare dal male le risorse indigene, a convertirle da potenza in atto, a rendere fertile quel che prima era infruttifero e selvaggio, se anche ricco di un'energia produttiva latente. Si vede subito, però, che il contraccambio reso dal suolo tebano al suo eroe civilizzatore si limita, fin qui, a ciò che qualsiasi terra senza storia, qualsiasi gente guerriera ancora priva di una struttura sociale potrebbe offrire al nobile pellegrino d'oltremare: una dimora ${ }^{8}$, un territorio da sfruttare, la protezione di un popolo in armi, legittimazione, potere.

Il secondo motivo è che un episodio centrale della storia di Cadmo, il suo sposalizio con Armonia, è contrassegnato proprio dalla presentazione di doni agli sposi da parte delle divinità ospiti. Sebbene i doni degli dei d'olimpo, quegli dei a così debole connotazione 'nazionale', abituati a visitare con uguale disinvoltura tutte le regioni del Mediterraneo, non appaiano immediatamente interpretabili come un'offerta del suolo e delle stirpi greche all'immigrato fenicio, c'è però una località, Samotracia, che nell'appropriarsi dell'episodio delle nozze di Cadmo in concorrenza con la tradizione tebana assegna a questi doni una evidente caratterizzazione locale. Nella versione raccolta da Diodoro Siculo (V, 48-49), che si ritiene derivata da tradizioni antiche ${ }^{9}$, Armonia non è la figlia di Ares e Afrodite, ma una figura indigena, figlia di Elettra e sorella di Iasione e Dardano,

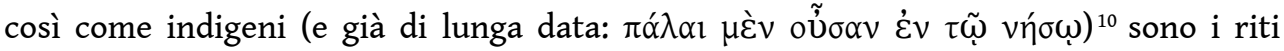
misterici a cui Cadmo viene iniziato prima delle nozze. L'isola di Samotracia diviene in questa occasione un grande centro d'irradiazione di civiltà, non solo a beneficio di Cadmo, ma dell'umanità tutta ${ }^{11}$, e in particolare delle vicine terre d'Asia, dove, racconta Diodoro, dopo il matrimonio vengono esportati i doni di natura più spiccatamente civilizzatrice: i misteri della Grande Madre, capaci di elevare moralmente gli uomini

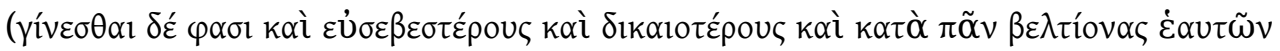

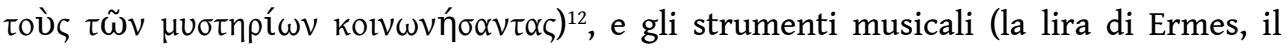
doppio aulo di Atena) ${ }^{13}$.

5 In questo episodio intravediamo la cosciente auto-rappresentazione di una Grecia più matura, o almeno di un frammento di essa (un'isola dell'Egeo settentrionale) che non ha da porgere allo straniero solo zolle da arare e uomini in armi in cambio dei suoi benefici, ma anche i frutti della religione misterica, della musica, di una moralità e di un'estetica progredite. È una Grecia non necessariamente successiva a quella desolata e feroce che Cadmo trova al suo arrivo in Beozia: il mito, almeno, non la presenta come tale; è bensì una Grecia più raffinata, culla essa stessa di civiltà nel momento in cui accoglie il civilizzatore venuto da fuori. La sua contrapposizione all'altra' Grecia può vedersi adombrata dal mito in forma di contrapposizione fra mondo olimpico (le divinità ospiti al matrimonio) e mondo ctonio (il drago, gli Sparti).

Nelle pagine che seguono sarà esplorato il rapporto fra la venuta di Cadmo e la risposta del substrato nativo con particolare riferimento agli episodi della fondazione di Tebe e delle nozze con Armonia.

\section{La fondazione di Tebe}

7 Tutta la vicenda di Cadmo, considerata sotto il profilo dei rapporti fra straniero e indigeno, può apparire a un primo sguardo come un vasto roveto di contraddizioni; e il 
suo viluppo più denso si situa forse negli avvenimenti che immediatamente precedono la fondazione di Tebe: in particolare, nelle relazioni fra Cadmo e gli Sparti.

8 Gli Sparti sono la progenie di un essere ferino e brutale che incarnava lo spirito primordiale del luogo; ma sono anche i futuri sudditi di Cadmo, la materia umana necessaria per costruire in quello stesso luogo una comunità civile. Essi nascono vestiti d'armi e subito violenti di una violenza cieca, che li travolge nella carneficina reciproca; ma nascono dai solchi come spighe di grano ${ }^{14}$, cioè come l'alimento che più di ogni altro simboleggia la domesticazione del suolo e il benessere di un'umanità già pacificata con la natura selvaggia.

i denti del drago, da cui gli Sparti germogliano, sono stati consegnati a Cadmo dagli dei (Atena o Ares, o ambedue) ${ }^{15}$, ciò che affratella Cadmo ad un altro eroe, Eeta, pupillo di Ares (metà dei denti, infatti, è riservata a lui) ${ }^{16}$; eppure il mito ci narra anche dell'ira di Ares contro lo stesso Cadmo e di un lungo castigo che l'eroe deve scontare per appagarla: ira e castigo di cui le fonti adducono motivazioni diverse, ma fra queste, appunto, anche l'uccisione del drago ${ }^{17}$.

Infine: in Euripide la terra tebana, dopo aver ammesso il sacrificio del drago (creatura

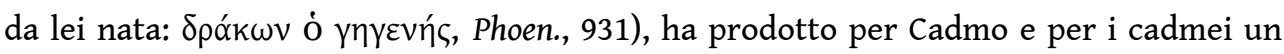
frutto di inestimabile valore, la «messe degli Sparti dal casco d'oro» (ibid., 939-940); tuttavia quella stessa terra, e per questi stessi motivi, è poi in collera col popolo di Tebe, reclama a distanza di sette generazioni ${ }^{18}$ un sacrificio espiatorio: «frutto in cambio di frutto, umano sangue in cambio di sangue» (ibid., 937-938). Dunque i suoi erano doni amari, che ancora dopo molto tempo esigono un contraccambio cruento: un

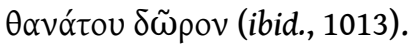

11 Dove sono allora i confini tra beneficio e ostilità? Come si dispongono le relazioni di amicizia e di lotta fra gli attori del mito? La terra, gli dei, sono alleati compiacenti o nemici caparbi? L'uccisione del drago che impediva a Cadmo di attingere acqua lustrale è un gesto di pietà, un'opera di bonifica, o è un 'peccato originale' che macchia la storia di Tebe ai suoi primordi?

12 Per dare una risposta a queste domande dobbiamo forse abbandonare l'impostazione sommaria che sovrappone le diverse fonti del mito, e le amalgama fra loro, sperando di ricavarne un quadro coerente. Dobbiamo invece tener presente che un mito, al suo stato, per così dire, crudo, non ancora filtrato da elaborazioni letterarie, non è necessariamente governato dalle stesse esigenze di coerenza e verosimiglianza psicologica che intervengono nei testi, e specialmente in particolari categorie di essi, come il racconto di un mitografo razionalizzante o la resa scenica di un poeta teatrale ${ }^{19}$. Il mito può relegare nel buio tutto ciò che è sfondo (perché gli Sparti appena venuti alla luce si sterminano a vicenda? che cosa, poi, trattiene i cinque destinati a sopravvivere - Udeo, Ctonio, Peloro, Iperenore, Echione - dall'aggredirsi l'un l'altro? ecc.); nel prestare ascolto ad un 'mito', infatti, noi siamo liberi di accordare spontaneamente la nostra fiducia alla sua logica fantastica, di accogliere così come si snoda il susseguirsi prodigioso dei fatti, e di sentirci esonerati dal commisurare quei fatti ai parametri psicologici e sociali, fisici e biologici, della realtà ordinaria.

13 In quanto mito di fondazione e insieme di legittimazione delle stirpi dominanti a Tebe in età storica, il mito degli Sparti esprime con straordinaria forza il valore dell'autoctonia e, a un tempo, il legame con un grande eroe civilizzatore venuto da fuori; esprime inoltre la vocazione guerriera, l'originario impulso divino (l'oracolo di 
Delfi) e, non ultimo, il senso del 'miracoloso', del 'magico' che sta alla radice di tutta la storia della città: e tanto basta al sentimento popolare così come all'orgoglio dei notabili di Tebe, all'estetica e al prestigio del loro potere. Sono invece gli autori letterari che, nel calare quel mito nella scena o nello sdipanare la catena delle cause e degli effetti in un racconto dettagliato, si scontrano con nodi d'incoerenza, e adottano ciascuno, per scioglierli, una propria chiave di lettura.

Diremo perciò che nel mito fondativo di Tebe (nella misura in cui sia possibile 'astrarre' un mito dalle sue testimonianze ${ }^{20}$ si fronteggiano forze antagoniste, ma non per il trionfo dell'una sull'altra e del 'bene' sul 'male', come in una sacra rappresentazione; bensì l'una sottopone l'altra a prove mortali e nello stesso tempo collabora con essa all'atto di nascita di un nuovo microcosmo, che è la città. In questo mescolarsi di lotta e di collaborazione non trova spazio - non è pertinente - il disagio della contraddizione.

Ma se spostiamo l'attenzione sulle voci concrete del mito, sulle testimonianze che ce lo consegnano, vediamo che le cose vanno diversamente. In Euripide, la contraddizione è deliberatamente cercata e approfondita, diviene alimento a una vicenda conturbante. La Terra ha donato, ma vuol essere gratificata per questo con nuovo sangue (Phoen., 931-941). L'atto di fondazione di Tebe si tramuta così in una ferita aperta: è la matrice di tutte le successive sventure della città ${ }^{21}$ (ibid., 1065-1066).

È proprio della forma mentale del drammaturgo raccogliere eventi molteplici entro un unico orizzonte di significato, dotare retrospettivamente di senso oggetti già apparsi sulla scena (come il chiodo del celebre aforisma di Čechov), o, com'è tipico in particolare dei tragici antichi, ricomporre in un'unica e robusta corda i trefoli sparsi di una vicenda che copre molte generazioni: ciò di cui le vicende degli Atridi e dei Labdacidi sono forse gli esempi più ovvi. Nelle Fenicie, Euripide pone al centro il guasto morale insanabile che si perpetua di generazione in generazione nella storia di Tebe, e di quel guasto ricaccia indietro le origini fino a Cadmo, così da poter interpretare l'intera storia della città in modo coerente, per circoli conchiusi di senso: la morte di Eteocle e Polinice chiude il cerchio che si era aperto con l'antica trasgressione di Laio; la morte di Meneceo quello che si era aperto con il sanguinoso gesto di Cadmo.

17 Nei mitografi invece la vicenda si fa razionalmente più limpida, ma in certo modo anche più povera. I nessi causali si ispessiscono a detrimento dei significati profondi: non sono più infatti la terra, il drago e il sangue versato a parlare simbolicamente il linguaggio del mito, ma sono fatti d'esperienza comune a spiegarci prosasticamente la storia. Per Ferecide ${ }^{22}$, ad esempio, Cadmo, vedendo sorgere dalla terra una moltitudine di uomini in armi, «si spaventa» ( $\delta \varepsilon i ́ \sigma \alpha \varsigma)$ e getta sassi contro di loro; quelli, credendosi

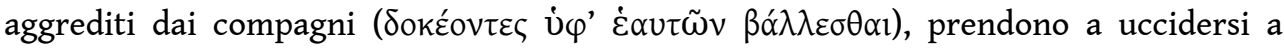
vicenda. In Apollodoro ${ }^{23}$ troviamo anche una spiegazione alternativa, ma di tenore simile: gli Sparti iniziano a massacrarsi, «gli uni venendo a contesa intenzionalmente ${ }^{24}$, gli altri senza sapere che cosa stessero facendo». Sebbene la distinzione tra sfida

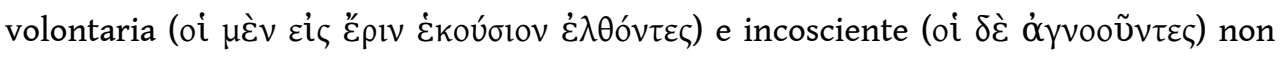
sia psicologicamente esaustiva, essa tradisce lo stesso bisogno di costruire una struttura causale e conferire al racconto una parvenza di plausibilità. Vediamo qui un mito 'imborghesito', in cui della grande frizione e fusione tra il forestiero e il nativo, o tra l'umano e il selvaggio, non restano che pallide tracce. 


\section{Le nozze con Armonia} Elettra $^{30}$, e non, «come si favolegga in Grecia» ${ }^{31}$, di Ares e Afrodite. I doni che gli sposi ricevono dagli dei meritano particolare attenzione. Con l'eccezione del monile e del peplo regalati ad Armonia da Atena, sono tutti, infatti, doni 'civili', molto meno destinati alla coppia nuziale che all'umanità intera: essi devono sprigionare la civiltà fra i popoli, propagandosi da Samotracia verso altri paesi. Diodoro li elenca: il grano di

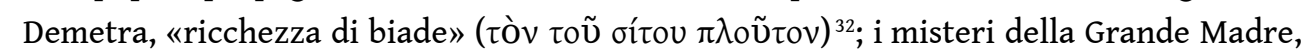
che in un secondo momento saranno trapiantati in Frigia col loro corredo di cimbali, timpani e cori orgiastici ${ }^{33}$; e gli strumenti musicali (lira ed aulo: Ermes e Atena li cedono agli umani), anche questi destinati a migrare verso l'Asia ${ }^{34}$. È una grande rivendicazione di potenza civilizzatrice.

Ma proprio gli ultimi, i doni musicali, ci invitano ad un trapasso alla versione tebana dello stesso episodio. Pindaro infatti, il più illustre rappresentante di quella versione ${ }^{35}$, è anche il grande valorizzatore del tema musicale nel mito di Cadmo. Come soltanto Peleo fra gli altri mortali, Cadmo ha goduto del privilegio sommo di udire la voce e le armonie degli dei: un privilegio ineffabile, per Pindaro, simbolico di tutte le fortune,

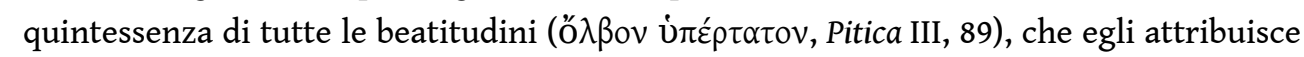
all'eroe nei suoi carmi non meno di tre volte (Pitica III, 88-91; Inno I, frr. 32 e 35c; cfr. Ditirambo II, 28-29). Anche nel racconto di Diodoro il matrimonio è onorato da un'offerta musicale di Apollo e delle Muse ${ }^{36}$; ma in Pindaro Cadmo riceve da Apollo un

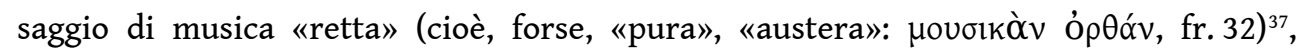
assiste alla danza delle Muse ( $\mu \varepsilon \lambda \pi$ ${ } \mu \varepsilon v \tilde{\alpha} v$, Pitica III, 90), ascolta il loro concento 
melodioso, «di fabbrica celeste» ( $\theta \varepsilon o ́ \delta \mu \alpha \tau o v ~ \kappa \varepsilon ́ \lambda \alpha \delta o v$, fr. 35c), mentre ripercorrono

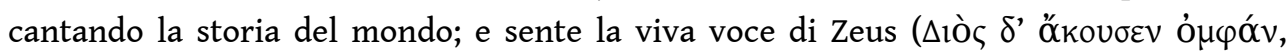
Ditirambo II, 29). Tutto il resto dei doni di nozze si riassume in un cenno brevissimo:

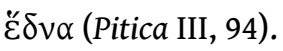

Ma questa musica divina udita da Cadmo alla sua festa di matrimonio «in Tebe dalle sette porte $»^{38}$ è un dono di Tebe stessa? Un omaggio della civiltà locale all'ospite forestiero? È la manifestazione di un'autocoscienza greca a fronte di un civilizzatore venuto di lontano? Certamente no: innanzi tutto perché Pindaro, diversamente da Bacchilide ${ }^{39}$, non accenna mai alle origini esotiche di Cadmo; poi perché gli dei discesi a Tebe dall'olimpo per onorare un eroe (proprio come in altra occasione discenderanno sul Pelio, in Tessaglia, per onorarne un altro: Pitica III, 90; Nemea V, 22-25) ${ }^{40}$ non esprimono certo la civiltà tecnica ed estetica di Tebe medesima, ma incarnano più semplicemente un livello superiore di esistenza, un loro proprio possesso di sovrumana sapienza, arte e bellezza. La cosmogonia che si dispiega nel canto delle Muse davanti a Cadmo nell'Inno I, non ha alcun 'color locale', è per l'appunto un grande racconto sull'universo: procede dall'antico connubio di Zeus e Temi alle miracolose vicende dell'isola di Delo, dalle nascite degli dei più giovani (Apollo, Atena) alla Titanomachia. Inoltre, Armonia qui è un'immortale, e Cadmo l'ha avuta dagli dei non come un ospite riceve doni dall'ospitante, né come un principe di altro paese è accolto per via matrimoniale in un casato locale; e neppure l'ha conquistata o rapita giungendo da straniero nel paese di lei, come nei racconti samotraci: bensì l'ha avuta per le sue

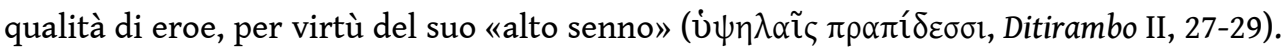
Si potrebbe in effetti dire che a nessun uomo, e quindi neanche a Cadmo, gli dei pindarici guardano come a un forestiero: essi guardano alle qualità umane, alla pietà, al coraggio, alla semenza, alle gesta ${ }^{41}$.

E tuttavia c'è un mortale, un tebano, che all'eroe fondatore della sua città ha da offrire un dono straordinario: questo tebano è Pindaro, il quale tacitamente ci invita a intendere le note dei suoi canti come un riflesso di quelle armonie celestiali ${ }^{42}$. È Pindaro che dispensa a Cadmo musica e gloria, come Omero le ha dispensate ad altri eroi, ad esempio a Nestore e a Sarpedone ${ }^{43}$. Benché questo rapporto tra Pindaro e Cadmo resti implicito, esso ci mostra una diversa possibile maniera di applicare il tema del dono e dello scambio al personaggio di Cadmo: non è l'orgoglio di una città civilizzatrice che qui prende corpo, bensì quello di un poeta, di un individuo, capace di restituire a Cadmo - come del resto a ogni altro grande eroe e a tutti i committenti delle sue odi fama imperitura a compenso della grandezza.

\section{Conclusioni}

L'interrogativo posto in partenza era se il fenicio Cadmo (o l'egiziano Cadmo, o il greco Cadmo reduce dal Vicino Oriente), che il mito raffigura nei panni di civilizzatore in terra greca, sperimenti a propria volta in Grecia un potere locale di civilizzazione, se cioè percepisca egli stesso l'influsso di una cultura morale e materiale diversa dalla propria (e tale influsso si traduca per lui in acquisizioni specifiche), o se invece l'opera civilizzatrice si compia tutta in un unico verso. La domanda è giustificata su un piano astratto da una considerazione di buon senso: l'archeologia ci dichiara senza equivoco che Tebe, come altre città-fortezza di età micenea, raggiunse un grado ragguardevole di sviluppo tecnico e organizzativo; ci suggerisce anche che essa intrattenesse un dialogo 
politico (fatto tra l'altro di doni reciproci) ${ }^{44}$ con i sovrani dell'Asia minore; se ne deduce come conseguenza probabile che i suoi contatti con le civiltà orientali abbiano avuto piuttosto la forma di un'impollinazione' reciproca, che di un mero assorbimento di stimoli da parte di un sostrato culturalmente avaro.

Ma la risposta alla domanda, per converso, non è in alcun modo scontata, dal momento che un mito ha tutte le ragioni per disinteressarsi dell'archeologia e per ignorare le ricostruzioni storiche del passato; il che vale a maggior ragione, com'è ovvio, in una civiltà prevalentemente orale come quella greca arcaica. Le analisi precedenti hanno mostrato, di fatto, che un'ambizione civilizzatrice autoctona nei confronti del visitatore straniero non si esprime nel mito di fondazione di Tebe, né nella versione tebana delle nozze di Cadmo e Armonia, ma solo nella tradizione samotracia di quelle nozze, a noi nota principalmente per il tramite di Diodoro Siculo. E a Samotracia tale ambizione si esprime con una certa perentorietà: nel racconto diodoreo (V, 48-49), a Cadmo non è accreditato nessun apporto alla cultura locale, mentre egli figura quasi come un personaggio di prestigio annesso da Samotracia alle proprie tradizioni per rivestirle di maggior lustro. Cadmo è un ospite insigne che ha ricevuto sull'isola gli splendidi doni di cui l'isola è capace, e che, al pari di Giasone e dei Dioscuri, di Eracle e di Orfeo ${ }^{45}$, ha tratto giovamento da quella polla di elevazione spirituale che sono i misteri della Grande Madre originari di Samotracia.

In altri contesti geografici e letterari il tema della reciprocità è magari più palese, o addirittura più urgente, ma assume connotati del tutto diversi da quelli di uno scambio fra civiltà. Dei generi letterari toccati nelle pagine precedenti, due lo mostrano in modo particolare: la tragedia attica (Euripide, Fenicie) e la lirica corale (Pindaro). Euripide incorpora il tema della reciprocità nella sua lettura problematizzante della storia di Tebe, ma lo fa trasmigrare da un piano culturale ad uno etico-sacrale, identificandolo col tema dello scambio di sangue con sangue (il sacrificio di Meneceo che sta a riparazione della morte del drago). In Pindaro, viceversa, la reciprocità che abbiamo intravisto è quella del dono, non della violenza, ma si situa sul livello astratto e metaforico dello scambio fra l'eroe e il cantore: fra lo splendore delle gesta e delle avventure, da un lato, e dall'altro il canto di cui quelle gesta hanno 'sete' ${ }^{\text {'46, }}$, perché vogliono essere da esso immortalate.

\section{BIBLIOGRAFIA}

ARAVANTINOS Vassilis L., Le scoperte archeologiche ed epigrafiche micenee a Tebe: un bilancio riassuntivo di un quinquennio (1993-1997) di scavi, in P. Angeli Bernardini (ed.), Presenza e funzione della città di Tebe nella cultura greca (Atti del convegno internazionale, Urbino, 7-9 luglio 1997), Pisa-Roma, Istituti editoriali e poligrafici internazionali, 2000.

BRESSON Alain, L'économie de la Grèce des cités, I: Les structures et les productions, Paris, Colin, 2007. CARRIÈRE Jean-Claude \& MASSONIE Bertrand, La Bibliothèque d'Apollodore (traduite, annotée et commentée), Paris, Les Belles Lettres, 1991. 
CASEVITZ Michel \& JACQUEMIN Anne, Diodore de Sicile. Bibliothèque historique, t. V (livre V), Paris, Les Belles Lettres, 2015.

CORDIANO Giuseppe \& ZORAT Marta, Diodoro Siculo. Biblioteca storica, vol. I (libri I-III), Milano, BUR, 2004.

EDWARDS Ruth B., Kadmos the Phoenician: A Study in Greek Legends and the Mycenaean Age, Amsterdam, A. M. Hakker, 1979.

FOWLER Robert L., Early Greek Mythography, I-II, Oxford, OUP, 2000/2013.

FRAZER James G., Apollodorus: The Library, I-II, London-New York, W. Heinemann \& G. P. Putnam's Sons, 1921.

GENTILI Bruno, Pindaro. Le Pitiche, a cura di B. Gentili, P. Angeli Bernardini, E. Cingano e

P. Giannini, Milano, Mondadori, $2000^{3}$.

GUETTEL COLE Susan, Theoi Megaloi: The Cult of Great Gods at Samothrace, Leiden, Brill, 1984.

HALL Edith, Inventing the Barbarian. Greek Self-Definition through Tragedy, Oxford, Oxford University Press, 1989.

MASTRONARDE Donald J., Euripides. Phoenissae (edited with introduction and commentary), Cambridge, Cambridge University Press, 1994.

MOST Glenn W., Postface: The Mazes of Mythography, in J. Pàmias (ed.), Apollodoriana: Ancient Myths, New Crossroads, Studies in Honour of F. J. Cuartero, Berlin-Boston, De Gruyter, 2017.

OLIVIERI Oretta, Miti e culti tebani nella Poesia di Pindaro, Pisa-Roma, Fabrizio Serra Editore, 2011.

PAPATHOMOPOULOS Manolis, Apollodori Bibliotheca, post Richardum Wagnerum recognita, Athina, Aletheia, 2010.

PARKE Herbert William \& WORMELL Donald Ernest Wilson, The Delphic Oracle, vol. II: The Oracular Responses, Oxford, Basil Blackwell, 1956.

PARKER Laetitia P. E., Euripides Alcestis, Oxford, Oxford University Press, 2007.

PORADA Edith, The Cylinder Seals Found at Thebes in Boeotia, «AOF», 28, 1981, pp. 1-70.

ROCCHI Maria, Kadmos e Harmonia. Un matrimonio problematico, Roma, L'«Erma» di Bretschneider, 1989.

SALVIAT François, SALAČ Antoine \& CHAPOUTHIER Fernand, Le théâtre de Samothrace, «BCH», 80, 1956, pp. 118-146.

SAVINO Ezio, Verso 'Quarta dimensione', introduzione a G. Ritsos, Quarta dimensione, Milano, Crocetti, 2013.

SCARPI Paolo \& CIANI Maria Grazia, Apollodoro. I miti greci, Milano, Mondadori, 1997.

VIAN Francis, Les origines de Thèbes : Cadmos et les Spartes, Paris, Klincksieck, 1963.

ZACCAGNINI Carlo, Lo scambio dei doni nel Vicino Oriente durante i secoli XV-XIII, Roma, Centro per le antichità e la storia dell'arte nel Vicino Oriente, 1973.

\section{NOTE}

1. Sulla questione dell'origine di Cadmo, dibattuta in antico come fra i moderni, vedi Edwards (1979) e, in sintesi, Olivieri (2011, 20-21, con bibliografia alla n. 15). 
2. Vian $(1963,104)$.

3. Vian (1963, 134-141).

4. Edwards (1979, 22-23; cfr. ibid., 32-33 per l'introduzione, ugualmente ascritta a Cadmo, di altre arti e tecniche, come la lavorazione dell'oro, l'estrazione mineraria, la costruzione di acquedotti). 5. Memorie di tali contatti sono racchiuse anche in altri miti, come quelli di Io, Danao, Europa. Sulle attestazioni archeologiche dei rapporti fra Tebe micenea e il Vicino Oriente (sigilli con iscrizioni cuneiformi e materiali preziosi di provenienza orientale nel tesoro della reggia di Tebe), vedi Aravantinos (2000, 32 e 42-44).

6. Porada (1981, 68-70) e Aravantinos (2000, 32-33).

7. Aravantinos (2000, 45-51).

8. Si noti che in alcune versioni del mito la domanda rivolta da Cadmo all'oracolo di Delfi non è dove si trovi sua sorella, ma dove egli stesso potrà insediarsi: schol. in Aesch. Sept. 486c (221, 7-8

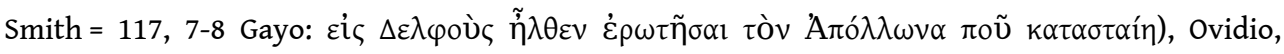
Metamorfosi, III, 8-9 (Phoebique oracula supplex / consulit et quae sit tellus habitanda requirit).

9. Forse, tra l'altro, da testi drammatici un tempo messi in scena a Samotracia, come quelli del tragediografo Dymas (TrGF, I, 130): vedi Salviat, Salač \& Chapouthier (1956, 142-145) e cfr. Vian $(1963,65$, n. 6).

10. Diodoro Siculo, Biblioteca storica, V, 48,4. Il testo di Diodoro riproduce qui e in seguito l'edizione di Casevitz \& Jacquemin (2015).

11. Sul valore universale e archetipico della festa di nozze offerta dagli dei, con la quale si fonda l'istituto del matrimonio e si introducono nel mondo alcune pratiche distintive del genere umano, come la cerealicoltura, vedi Rocchi $(1989,83-86)$.

12. Diod. Sic., V, 49, 6.

13. Ibid., 49, 4 .

14. Euripide, Eracle, 5; Fenicie, 939; Baccanti, 264, 1026, 1315.

15. Atena: Euripide, Fenicie, 667 (per il testo vedi Mastronarde, 1994, 341-342), Apollonio Rodio, Argonautiche, III, 1183-1187, e cfr. Stesicoro, F 96 Finglass; Ares: Ellanico, FGrHist, I, 4 F 1a (dove tuttavia Fowler, 2000, accoglie l'emendamento di 'A $\rho \varepsilon \omega \varsigma$ in A A $\eta v \tilde{\alpha} \varsigma)$, e cfr. Euripide, Eracle, 252-253; Atena ed Ares insieme: Ferecide, FGrHist, I, 3 F 22ab.

16. Ferecide, FGrHist, I, 3 F 22ab, Apollonio Rodio, Argonautiche, III, 1184; cfr. Vian (1963, 26).

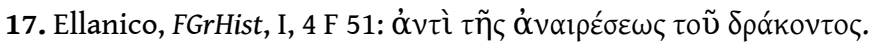

18. Meneceo il giovane, che dovrà dissetare la terra col suo sangue per estinguerne l'ira, è nipote di Meneceo il vecchio, nipote di Penteo, nipote di Cadmo.

19. Cfr. le considerazioni di Parker (2007, XIv) a proposito del mito di Alcesti: «in drama, where the hero walks and talks before our eyes, questions of motivation and character become inescapable»; o quelle - per passare a un ambito letterario tutto moderno - di Savino $(2013,16)$ a proposito della poesia di Ghiannis Ritsos, dove il mito antico viene immerso nella quotidianità e come estenuato in dettagli psicologici privati, stremato in soliloqui di persone vive e pensanti: «il mito si estingue. Entra la vita con la sua pienezza ingombrante».

20. Questa forma 'cruda' e impersonale del mito può identificarsi con ciò che Most $(2017,228)$ denomina «le antiche storie in sé» («the ... ancient stories themselves»), situate al cuore del grande dedalo della mitologia e mitografia: ai margini del dedalo sta la ricerca scientifica moderna, che applica metodi razionali allo studio di quelle antiche storie; mentre fra cuore e periferia si collocano le elaborazioni letterarie e le compilazioni mitografiche degli antichi stessi.

21. «The founding act that began the recurrent troubles» (Mastronarde, 1994, 434; cfr. ibid., 30).

22. FGrHist, I, 3 F $22 \mathrm{ab}$.

23. Biblioteca, III, 4, 1 [23].

24. Con غ́koúøiov, lezione preferita da Carrière \& Massonie (1991), Scarpi (1997) e Papathomopoulos (2010); v.l. ókoúøıov, «loro malgrado», accolta da Frazer (1921). 
25. Nonno, Dionisiache, XIII, 349-366. La versione libica è collegata a entrambe le precedenti: a quella samotracia perché la Libia è patria di Atlante, il nonno materno di Armonia (cfr. Vian, 1963, 62, n. 6); a quella tebana perché le nozze in Libia preludono alla celebrazione più solenne che si terrà in Tebe, e che Nonno ha già descritto nel quinto libro (88-210).

26. Sulle nozze libiche, vedi Rocchi (1989, 73-81).

27. Ellanico, FGrHist, I, 4 F 28; cfr. inoltre Mnaseas, FHG, III, 154, fr. 23. Altri testimoni della versione samotracia, come Eforo (FGrHist, II A, 70 F 120) e Arriano (FGrHist, II B, 156 F 64), parlano di un rapimento di Armonia ad opera di Cadmo, e di feste locali che rievocano quell'evento in forma di dramma sacro.

28. Sulla religione e i culti di Samotracia, vedi Guettel Cole (1984).

29. Diod. Sic., V, $48,4$.

30. Che Armonia, nella tradizione che la fa originaria di Samotracia, sia un'eroina mortale e non una dea, è deducibile in Diodoro dallo statuto mortale di suo fratello Iasione (Diod. Sic., V, 49, 2) e sottolineato da Nonno con l'attributo ĖंıxӨovín (Dionisiache, XIII, 340). Sulla stretta associazione di Elettra a Samotracia, vedi Apollonio Rodio, Argonautiche, I, 916, Valerio Flacco, Argonautiche, II, 431, e Casevitz \& Jacquemin $(2015,250)$.

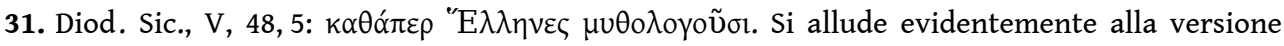
continentale del mito. Sul valore di $\mu \cup \theta 0 \lambda$ oүé $\omega$ in Diodoro, vedi Cordiano $(2004,44)$.

32. 49, 1 e 4. Sulla cerealicoltura come la prima fonte di ricchezza in Grecia, vedi Bresson (2007, 124-128).

33. $49,1-2$ e 6 .

34. 49, 1 e 4. Sul significato culturale dei doni musicali, vedi Rocchi (1989, 87-107).

35. Un altro testimone è Apollodoro (Biblioteca, III, 4, 2 [25]); Pausania (IX, 12,3) menziona il luogo dell'agora di Tebe nel quale ancora ai suoi giorni si additavano i resti del talamo di Cadmo e Armonia, nonché il luogo, pure nell'agora, dove si diceva che avessero cantato le Muse.

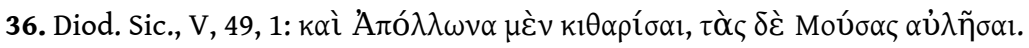

37. Sulla collocazione e sul possibile significato di questo frammento (incluso in Parke \& Wormell, 1956, 62-63), vedi Vian (1963, 27-28) e Rocchi $(1989,114)$.

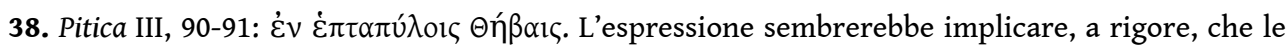
nozze si celebrino quando Tebe è già stata fondata e munita di fortificazioni, quando cioè ha già una fisionomia urbana caratteristica ed è potenzialmente capace di esprimere una cultura locale. Ma forse l'aggettivo $\dot{\varepsilon} \pi \tau \alpha \pi \dot{\lambda} \lambda o r \varsigma$ ha qui soltanto un valore decorativo.

39. Ditirambo XIX, 46-48.

40. Si tratta delle nozze di Peleo e Teti.

41. Così anche Peleo è premiato dagli dei con una sposa immortale, Teti, per riguardo alla sua

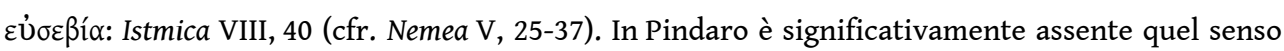
di un'opposizione fra Grecità e Oriente che viene costruendosi nell'età delle guerre persiane (Hall, 1989), quando la Grecia viene a confrontarsi in modo violento con una civiltà linguisticamente, culturalmente e politicamente diversa, a saggiare teatri di guerra remoti (come l'Egitto) e a sviluppare per reazione un più vigoroso senso della propria identità.

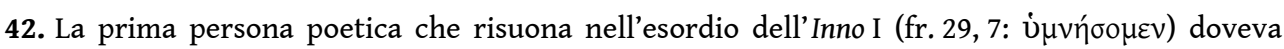
riaffiorare in altre parti dell'ode, e certamente dev'essere intesa come l'implicito termine di paragone terreno per Apollo e per le Muse: cfr. il famoso proemio della Pitica I, con Gentili $(2000,12)$.

43. Pitica III, 112-115.

44. Sugli aspetti ideologici e specialmente politici del dono nel Mediterraneo orientale antico, vedi Zaccagnini (1973).

45. Diod. Sic., V, 49, 6. 


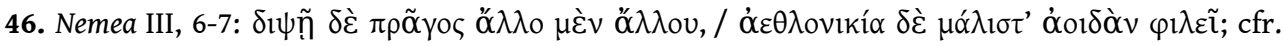

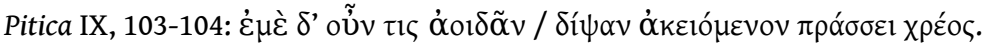

\section{RIASSUNTI}

Cadmo, straniero proveniente dalla Fenicia, offre alla Grecia dei doni inestimabili, quali dei nuovi culti, l'uccisione del drago e l'alfabeto. Ma che cosa ha da offrirgli in cambio la Grecia? La domanda può essere posta in altri termini: il mito di Cadmo include una qualche idea di reciprocità? I benefici provenienti dall'esterno - e più specificamente dal Vicino Oriente - sono semplicemente accolti dai Greci con ammirazione e gratitudine, oppure i Greci stessi hanno da rivendicare una loro propria capacità di civilizzazione? Questo articolo si basa sul presupposto secondo il quale gli scambi culturali tra due civiltà non sono mai monodirezionali, e esplora tale questione attraverso l'analisi di due episodi del mito di Cadmo: la fondazione di Tebe e il matrimonio di Cadmo con Armonia.

Cadmos offre à la Grèce des cadeaux inestimables, tels de nouveaux cultes, le meurtre du dragon et l'alphabet. Mais qu'est-ce que la Grèce a à lui offrir en échange ? En d'autres termes, le mythe de Cadmos exprime une quelconque forme de réciprocité ? Les bénéfices provenant de l'étranger - et plus spécifiquement du Proche-Orient - sont-ils juste reçus par les Grecs avec admiration et gratitude, ou alors les Grecs eux-mêmes revendiquent-ils leur propre capacité de civilisation? Cet article se fonde sur l'idée selon laquelle les échanges culturels entre deux civilisations ne sont jamais mono-directionnels, et explore cette question à travers l'analyse de deux épisode du mythe de Cadmos : la fondation de Thèbes et son mariage avec Harmonie.

Cadmus, a foreigner from Phoenicia, brings invaluable gifts to Greece, such as new cults, the slaying of the dragon, and the alphabet. But what does Greece can offer him in return? To say it differently: does the myth of Cadmus include any idea of reciprocity? Are benefits from abroador, more specifically, from the Near East-merely received by the Greeks with awe and gratitude, or do the Greeks themselves lay claim to having their own capacity to civilize? This article takes as its premise the idea that cultural exchanges between two civilizations are never entirely monodirectional, and explores this issue with special reference to two episodes of the myth: Cadmus' foundation of Thebes and his marriage with Harmonia.

\section{INDICE}

Mots-clés : Cadmos, Harmonie, Spartes, don

Parole chiave : Cadmo, Armonia, Sparti, dono

Keywords : Cadmus, Harmonia, Spartoi, gift 


\section{AUTORE}

\section{ANNAMARIA PERI}

Ludwig-Maximilians-Universität München.

Annamaria Peri est chercheuse à la Ludwig-Maximilians-Universität de Münich. Elle a obtenu son diplôme à l'École normale de Pise. Ses domaines privilégiés de recherche sont la poésie lyrique chorale d'époque tardo-archaïque (Pindare), Homère et la métrique grecque. 